\title{
Risk assessment of oil price from static and dynamic modelling approaches
}

Zhi-Fu Mi a,b, Yi-Ming Wei b,c,d,*, Bao-Jun Tang b,c,d,*, Rong-Gang Cong e,f,", Hao Yu ${ }^{\text {b,c,d }}$, Hong Cao ${ }^{\text {b,c,d }}$, Dabo Guan ${ }^{\text {a,b }}$

a School of International Development, University of East Anglia, Norwich NR4 7TJ, UK

${ }^{\mathrm{b}}$ Center for Energy and Environmental Policy Research, Beijing Institute of Technology, Beijing 100081, China

c School of Management and Economics, Beijing Institute of Technology, Beijing 100081, China

${ }^{\mathrm{d}}$ Collaborative Innovation Center of Electric Vehicles in Beijing, Beijing 100081, China

${ }^{\mathrm{e}}$ Department of Environmental Science, Aarhus University, Roskilde 4000, Denmark

${ }^{\mathrm{f}}$ Centre for Environmental and Climate Research (CEC), Lund University, Lund S-22362, Sweden

\begin{abstract}
The price gap between West Texas Intermediate (WTI) and Brent crude oil markets has been completely changed in the past several years. The price of WTI was always a little larger than that of Brent for a long time. However, the price of WTI has been surpassed by that of Brent since 2011. The new market circumstances and volatility of oil price require a comprehensive re-estimation of risk. Therefore, this study aims to explore an integrated approach to assess the price risk in the two crude oil markets through the Value at Risk (VaR) model. The VaR is estimated by the extreme value theory (EVT) and GARCH model on the basis of Generalized Error Distribution (GED). The results show that EVT is a powerful approach to capture the risk in the oil markets. On the contrary, the traditional Variance-Covariance and Monte Carlo approaches tend to overestimate risk when the confidence level is $95 \%$, but underestimate risk at the confidence level of $99 \%$. The VaR of WTI returns is larger than that of Brent returns at identical confidence levels. Moreover, the GED-GARCH model can estimate the downside dynamic VaR accurately for the WTI and Brent oil returns.
\end{abstract}

\section{Acknowledgements:}

The authors gratefully acknowledge the financial support of National Natural Science Foundation of China under the Grant Nos. 71020107026 and 71273031.

* Corresponding author: E-mail: $\underline{\text { rc@envs.au.dk }(R o n g-G a n g ~ C o n g)}$ 


\section{Risk assessment of oil price from static and dynamic modelling approaches}

Abstract: The price gap between West Texas Intermediate (WTI) and Brent crude oil markets has been completely changed in the past several years. The price of WTI was always a little higher than that of Brent for a long time. However, the price of WTI has been surpassed by that of Brent since 2011. The new market circumstances and volatility of oil price require a comprehensive re-estimation of risk. Therefore, this study aims to explore an integrated approach to assess the price risk in the two crude oil markets through the Value at Risk (VaR) model. The VaR is estimated by the extreme value theory (EVT) and GARCH model on the basis of Generalized Error Distribution (GED). The results show that EVT is a powerful approach to capture the risk in the oil markets. On the contrary, the traditional Variance-Covariance and Monte Carlo approaches tend to overestimate risk when the confidence level is $95 \%$, but underestimate risk at the confidence level of 99\%. The VaR of WTI returns is larger than that of Brent returns at identical confidence levels. Moreover, the GED-GARCH model can estimate the downside dynamic VaR accurately for the WTI and Brent oil returns.

Keywords: Value at risk; GED-GARCH; Extreme value theory; Risk quantification; oil markets

JEL Classification: C13; G32; Q40

\section{Introduction}

Oil plays an important role in the production and life, so crude oil price is usually taken as an indicator for the world economic situation. Meanwhile, the volatility of oil price has an effect on socioeconomic system (Ji and Guo 2015; Cong and Shen 2013). The frequent volatility of oil prices lead to great extreme market risk. It has brought huge potential losses to oil market 
stakeholders and participants (Zhang and Wei 2010). Therefore, the measurement of oil price risk has become one of critical issues for both academicians and oil market participants. In addition, the risk of oil price is differential for various participants, and both upside and downside risk exist in markets. Specifically, the buyers have losses when the oil price increases. On the contrary, the sellers occur losses if the oil price declines.

Currently, the downside risk of oil price has aroused great concern for two reasons. First of all, the oil prices have decreased since 2014 (figure 1). The average spot prices of crude oil in West Texas Intermediate (WTI) and Brent were 97.98 and 108.56 dollars per Barrel, respectively, in 2013. They both decreased to less than 30 dollars per Barrel in early 2016. Second, the price gap between WTI and Brent oil markets has been completely changed. For a long time, the oil prices of two markets were quite close, and the price of WTI was a little higher than that of Brent. However, this relationship has been completely changed since 2011 (figure 1). In other words, the price of WTI has been surpassed by that of Brent.

<Figure 1 is inserted around here.>

To date, many risk measurement techniques and models have been developed in the literature (Zhang and Wei 2011; Aloui and Mabrouk 2010). Value at risk (VaR), initially suggested by Baumol (1963), provides recapitulative and comprehensive advantages in estimating extreme risk in markets. We choose VaR to estimate the price risk of oil markets because it is a good measure of the downside risk (Boroumand and Zachmann 2012). The VaR can be divided into static and dynamic VaR. The former is a single real number which demonstrates market risk using the probability distribution of random variables. The dynamic VaR which estimates the risk with a series of fluctuating values demonstrates the risk characteristics over time. 
Many methods have been developed to calculate VaR during the past several decades. However, two limitations exist in these methods. First of all, most of the approaches only focused on the static or dynamic VaR. The common methods of calculating static VaR are Variance-Covariance, Monte Carlo approaches, historical simulation, and RiskMetrics (Giot and Laurent 2003). The dynamic VaR is usually analyzed by time series methods (e.g. GARCH) (Chang 2011; Chuang et al. 2015). To the best of our knowledge, a few studies have estimated both static and dynamic VaR using an integrated model. Second, most methods use the assumption of standard normal distribution. However, a great deal of empirical research has demonstrated that the assumption fails in oil markets (Fan et al. 2008).

Therefore, this study aims to explore an integrated approach to measure both static and dynamic VaR in oil markets and compare the risk characteristics in WTI and Brent. To be specific, the VaR is estimated by the extreme value theory (EVT) and GARCH model on the basis of Generalized Error Distribution (GED). The method is applied to assess the risk in the WTI and Brent oil markets. The rest of the paper is organized as follows. Section 2 is the literature review. Section 3 introduces the method of calculating VaR. Section 4 shows the empirical results and discussions, while Section 5 provides concluding remarks.

\section{Literature review}

To date, many risk measurement techniques and models have been developed to quantify oil price extreme risk. VaR has become a widely used tool to estimate market risk (Parkinson and Guthrie 2014; Ze-To 2012). Mi and Zhang (2011) employed VaR to estimate risk for the European Union Emissions Trading Scheme and compare its downside and upside risk. Bianconi and Yoshino (2014) calculated the VaR based on the GARCH model with Student's t distribution for the errors. 
It was used to analyze sixty-four oil and gas companies in twenty-four countries. Reboredo and Rivera-Castro (2014a) utilized VaR to estimate the risk of gold markets for different time investment horizons. Silva Filho et al. (2014) applied VaR to estimate the risk in the broad stock market indexes from four countries, including Britain, the United States, Mexico and Brazil.

With the development of VaR modeling techniques, some literature began to compare the performance of different calculating methods in risk management (Sadeghi and Shavvalpour 2006; Lee, Chiu, and Cheng 2010). Cabedo and Moya (2003) estimated VaR of oil price using standard historical simulation method, historical simulation method based on ARMA forecasts and variance-covariance method. They showed that historical simulation method based on ARMA forecasts was more flexible to estimate VaR. He et al. (2012) developed a multivariate wavelet denoising method to estimate the VaR in crude oil markets. The results showed that the developed method outperformed the DCC-GARCH approach and Exponential Weighted Moving Average approach. Su (2014) utilized four GARCH-based models to explore which method is more appropriate to estimate VaR based on the accuracy estimation. Empirical results showed that the SGED performed better than the normal and GED distribution for a long position. Huang (2015) used the threshold stochastic volatility method to assess the VaR for stock indices. The results showed that this method is more reliable than others.

EVT approach has been widely used for risk estimation in many fields, such as insurance (McNeil 1997), finance (Ergen 2014), hydrology (Katz, Parlange, and Naveau 2002), and energy market (Nomikos and Pouliasis 2011). It is an appropriate method to research the behaviors in extreme observations. EVT is focused on the tails of one distribution, while traditional approaches usually concentrate on the whole distribution. It has been proved to be an appropriate method to 
analyze the risk in the fields where the extreme values appear frequently (Fretheim and Kristiansen 2015).

In this paper, we use EVT to measure the static VaR in WTI and Brent crude oil market and compare the results with the traditional approaches. Moreover, GED-GARCH model is used to estimate the dynamic VaR, because of the significant volatility clustering in the WTI and Brent return series. The static and dynamic VaR of oil prices are estimated in an integrated framework, and the risk characteristics in WTI and Brent are compared.

\section{Methodology}

\subsection{Framework of EVT-GED-GARCH integrated model}

The VaR in Brent and WTI oil markets is estimated using the EVT-GED-GARCH integrated approach which combines EVT and GED-GARCH (figure 2). First, the static VaR is estimated by three methods, including EVT, Variance-Covariance, and Monte Carlo. The three methods' performance in VaR estimation is assessed by the the likelihood ratio (LR) test. Second, the dynamic VaR is estimated by GARCH-type model based on the GED. Finally, this study compares the crude oil risk between the WTI and Brent markets, and estimates the upside and downside risk.

$<$ Figure 2 is inserted around here.>

\subsection{Definition of VaR}

Let the crude oil price on day $t$ be $C_{t}$. The returns are defined as (Cong et al. 2008)

$$
R_{t}=100\left(\ln C_{t}-\ln C_{t-1}\right) .
$$

Analogously, the losses are defined as $100\left(\ln C_{t-1}-\ln C_{t}\right)$. We use the return series and loss series to calculate the upside and downside VaR, respectively. This study estimates the upside VaR as well as downside VaR. The following methodology is used to calculate upside VaR, and 
downside VaR can be gotten from the loss series in the same way.

VaR is a threshold loss value for a given portfolio and time horizon at a confidence level (Duffie and Pan 1997). Suppose $F(r)$ is the distribution function of oil returns and $p$ is the confidence level of VaR, then upside VaR can be defined as

$$
\mathrm{P}\left(R \leq V a R_{p}\right)=p, \quad \operatorname{VaR}_{p}=F^{-1}(p) .
$$

\subsection{Static VaR based on EVT}

EVT was one of methods to deal with a generalized extreme value distribution which was brought up by Jenkinson (Embrechts, Klüppelberg, and Mikosch 1997). Peaks over threshold approach is usually used to focus the largest events as well as these events which are larger than some threshold (McNeil and Frey 2000). In this paper, $\left\{R_{t}\right\}$ refers to the distribution function of oil return series,

$$
F(r)=\mathrm{P}\left(R_{t} \leq r\right)
$$

The distribution of excess values of return series which is over a given threshold $u$ is

$$
F_{u}(y)=\mathrm{P}(R-u \leq y \mid R>u)=\frac{F(y+u)-F(u)}{1-F(u)}, y>0 .
$$

The distribution $\left(F_{u}(y)\right)$ can be estimated by the Generalized Pareto Distribution (GPD) when the threshold is sufficiently large (Pickands 1975; Balkema and De Haan 1974),

$$
G(y)=\left\{\begin{array}{ll}
1-\left(1+\xi \frac{y}{\theta}\right)^{-1 / \xi} & \text { if } \xi \neq 0 \\
1-e^{-y / \theta} & \text { if } \xi=0
\end{array},\right.
$$

where $\xi$ and $\theta$ are the shape parameter and scale parameter, respectively. Suppose $y=r-u$, the estimation of tail can be gotten as

$$
F(r)=(1-F(u)) G(y)+F(u) .
$$

The empirical cumulative distribution function (CDF) is applied to estimate the $F(u)$. 
Suppose the exceedances' number is $N$ and the sample size is $n$, then the distribution of CDF

is

$$
F(u)=\frac{n-N}{n}
$$

Therefore, the estimate of $F(r)$ can be obtained as

$$
\overline{F(r)}=1-\frac{N}{n}\left(1+\bar{\xi} \frac{r-u}{\bar{\theta}}\right)^{-1 / \bar{\xi}}
$$

where $\bar{\theta}$ and $\bar{\xi}$ are the estimates of the $\theta$ and $\xi$, respectively. They are gotten from the estimation of Eq. (5). VaR is the p-quantile of the distribution for a given confidence level $p$.

Therefore, VaR can be calculated by

$$
V a R=u+\frac{\bar{\theta}}{\bar{\xi}}\left(\left(\frac{n}{N}(1-p)\right)^{-\bar{\xi}}-1\right)
$$

The threshold is one of key parameters in this approach. It is usually obtained via the Mean Excess Function. Its definition is

$$
e(u)=\frac{\sum_{t=1}^{n}\left(R_{t}-u\right)}{\sum_{t=1}^{n} 1_{\left\{R_{t}>u\right\}}},
$$

where $1_{\{\bullet\}}$ is the indicator function. If the Mean Excess Function is a straight line above the threshold, the data follows the Generalized Pareto Distribution (Mi and Zhang 2011).

\subsection{Dynamic VaR based on GARCH}

In this paper, GARCH model is used to estimate the dynamic VaR of oil price. It can be presented as (Bollerslev 1986)

$$
\begin{gathered}
R_{t}=X_{t}^{\prime} \beta+\varepsilon_{t}, \\
h_{t}=\alpha_{0}+\sum_{i=1}^{l} \alpha_{i} \varepsilon_{t-i}^{2}+\sum_{j=1}^{m} \beta_{j} h_{t-j},
\end{gathered}
$$

where $R_{t}$ refers to the oil price returns, and $X_{t}$ refers to a vector which include independent 
variables, and $\beta$ refers to the coefficient vector.

Traditional literature usually use estimate extreme risk under the assumption of normal distribution. It is not appropriate in the oil market, because the returns and losses of oil price often have fat tails and leptokurtic distributions. In this paper, GED is applied to assess the residual series of GARCH models (Nelson 1990). Its probability density function is

$$
f(\varepsilon)=\frac{k * \exp \left(-\frac{1}{2}|\varepsilon / \lambda|^{k}\right)}{\lambda * 2^{[(K+1) / k]} \Gamma(1 / k)} \quad(0 \leq k \leq \infty),
$$

where $\lambda=\left[\frac{2^{(-2 / k)} \Gamma(1 / k)}{\Gamma(3 / k)}\right]^{1 / 2}, k$ refers to freedom degree, and $\Gamma(\bullet)$ refers to gamma function (Fan et al. 2008). Therefore, the dynamic VaR is gotten by

$$
\operatorname{VaR}_{t}=\mu_{t}+z_{p} \sqrt{h_{t}}
$$

where $\mu_{t}$ and $h_{t}$ refer to conditional expectation and conditional variance, respectively, and $z_{p}$ refers to $p$-quantile of the Generalized Error Distribution.

\subsection{Evaluation of VaR models}

Over the last few decades, several methods have been proposed for testing the reliability of VaR results, such as the likelihood ratio (LR) test developed by Kupiec (1995), the Markov test developed by Christoffersen (1998), the duration-based test developed by Christoffersen and Pelletier (2004), and the multilevel test developed by Leccadito et al. (2014). This paper utilizes the LR test which is the most widely used approach.

Based on the LR test developed by Kupiec (1995), the $L R$ is defined as

$$
L R=-2 \ln \left[p^{n-N}(1-p)^{N}\right]+2 \ln \left[(1-f)^{n-N} f^{N}\right]
$$

where $p$ refers to the level of confidence, $N$ refers to the failure times, $n$ refers to the sample size, and $f$ refers to the failure rate. Therefore, the expected failure rate is $1-p$, and the null hypothesis is $H_{0}: 1-p=f$. 
The $L R$ follows $\chi^{2}$ distribution and its degree of freedom is one under the null hypothesis condition. Its critical values are 3.84 and 6.64 when the confidence level is $95 \%$ and $99 \%$, respectively. If $L R$ is smaller than the critical values, the null hypothesis is accepted, which means that the estimation of $\mathrm{VaR}$ is reliable. Otherwise, the VaR model is not reliable.

\section{Result analysis and discussions}

\subsection{Data sources}

In this paper, the daily spot crude oil prices in Brent and WTI markets from May 20th 1987 to December 31st 2014 are used. They are obtained from the Energy Information Administration of America. There are 7069 daily return samples which are presented in figure 3.

<Figure 3 is inserted around here.>

Table 1 shows the basic statistics of price returns in Brent and WTI markets. It can be seen that both means and standard deviations of the two returns are in the neighborhood, and the standard deviation of the WTI is a little bit bigger than that of Brent. In addition, both price returns have fat left tail and leptokurtic distribution, because kurtoses are greater than three and the skewnesses are negative. The Jarque Bera (JB) test also verifies that the oil price returns do not obey the standard normal distribution (Reboredo and Rivera-Castro 2014b).

<Table 1 is inserted around here.>

\subsection{Static VaR models estimation and test}

In the process of calculating the VaR based on EVT, the threshold value $u$ is one key parameter which is very subjective and controversial (Marimoutou, Raggad, and Trabelsi 2009). The Mean Excess Function (MEF) is used to determine the threshold value. If the Mean Excess Function is a straight line above the threshold, the data follows the Generalized Pareto Distribution. Hence, the 
appropriate threshold is the value above which the MEF appears approximately linear (Mi and Zhang 2011).

The WTI and Brent loss series are taken as examples, and their MEF plots are shown in the figure 4 and figure 5, respectively. In the MEF plot of WTI loss series, the threshold value is set to 3.0, above which $e(u)$ appears about linear. For the same reason, the threshold of Brent loss series is set to 3.6.

$<$ Figure 4 is inserted around here.>

$<$ Figure 5 is inserted around here.>

The upside and downside VaR in Brent and WTI oil price returns are obtained on the basis of the thresholds discussed above. Both $95 \%$ and $99 \%$ are chosen as the confidence levels. This paper also use the Monte Carlo (MC) and Variance-Covariance (VC) methods to estimate the VaR in order to compare the EVT approach and traditional methods. The results of static VaR are reported in table 2 and table 3, and the $L R$ values of three methods are demonstrated in figure 6 .

<Table 2 is inserted around here.>

$<$ Table 3 is inserted around here.>

$<$ Figure 6 is inserted around here.>

It can be obtained that the null hypotheses of EVT-VaR may be accepted, because its $L R$ values are all smaller than the critical values. It reveals that the VaR on the basis of the EVT approach is statistically reliable for Brent and WTI markets during the sample period. On the contrary, the $L R$ values of VaR using $\mathrm{VC}$ and $\mathrm{MC}$ approaches are mostly larger than the critical values, which reveals that traditional approaches may misestimate the VaR in WTI and Brent crude oil market. Therefore, EVT is a better approach to assess the VaR of oil prices in the Brent and WTI markets 
compared to the $\mathrm{VC}$ and $\mathrm{MC}$ approaches.

Traditional approaches tend to overestimate risk at the $95 \%$ confidence level but underestimate risk at the $99 \%$ confidence level for the oil price returns. Table 2 shows that the VaR based on the $\mathrm{VC}$ and $\mathrm{MC}$ are larger than EVT-VaR, which results in that failure rates of $\mathrm{VC}$ and $\mathrm{MC}$ approaches are much less than 5\%. On the contrary, the VaR based on the VC and MC approaches are less than EVT-VaR when the confidence level is $99 \%$ (table 3). This phenomenon is caused by the fat tail of WTI and Brent returns.

No matter for upside or downside, the VaR of WTI returns is larger than that of Brent returns at identical confidence levels (95\% or 99\%). It demonstrates that WTI crude oil market has more extreme risks compared to Brent market.

\subsection{Dynamic VaR models estimation and test}

This paper applies the GED-GARCH model to calculate the dynamic VaR of the Brent and WTI oil returns, because the returns have significant volatility and do not follows the normal distributions. There are two requirements in the determination of GARCH model. First, the coefficients are positive and significant. Second, the AIC value is relatively low. We test the $\operatorname{GARCH}(2,2), \operatorname{GARCH}(2,1), \operatorname{GARCH}(1,2)$ and $\operatorname{GARCH}(1,1)$ models. The $\operatorname{GARCH}(1,1)$ is finally chosen according the two requirements. As shown in table 4, GED degrees of freedom of WTI and Brent returns are 1.3235 and 1.3525 , respectively. They are both smaller than two, which verifies that the returns have fat tails than standard normal distribution.

<Table 4 is inserted around here.>

Using the GED-GARCH model, we assess the downside and upside dynamic VaR in the two oil markets. The risk at $95 \%$ and $99 \%$ confidence levels are both measured. The performance of 
GED-GARCH based dynamic VaR is shown in table 5 .

$<$ Table 5 is inserted around here.>

According to the table 5, the GED-GARCH model can reliably estimate the downside dynamic VaR of Brent and WTI oil price returns. The table 5 demonstrates that all $L R$ values of downside VaR are smaller than the critical values. It reveals GED-GARCH model is reliable to assess the downside VaR. But most $L R$ values of upside VaR are bigger than the critical values. So the GED-GARCH model fail to estimate the upside extreme risk during the sample period. However, both downside and upside VaR of Brent returns are statistically reliable at $99 \%$ confidence level, which is shown in figure 7.

$<$ Figure 7 is inserted around here.>

\section{Conclusions and remarks}

This study utilizes the EVT-GED-GARCH approach to measure the static and dynamic VaR in the WTI and Brent oil markets during the period of 1987-2014. The static VaR is estimated by three methods, including EVT, Variance-Covariance, and Monte Carlo. The three methods' performance in VaR estimation is assessed by the LR test. Then, the dynamic VaR is estimated by GED-GARCH. Several conclusions can be drawn as follows.

(1) EVT is reliable to assess the static VaR for price returns in Brent and WTI markets. Compared to the traditional methods (Monte Carlo and Variance-Covariance), EVT can estimate the VaR more accurately for oil price returns. All the $L R$ values of EVT-VaR are strictly smaller than the critical values, while all the $L R$ values of VaR using VC and MC approaches are larger than critical values.

(2) Traditional approaches tend to overestimate risk at the $95 \%$ confidence level but 
underestimate risk at the $99 \%$ confidence level. To be specific, the VaR based on the VC and MC are larger than EVT-VaR when the confidence level is $95 \%$, which results in that failure rates of $\mathrm{VC}$ and $\mathrm{MC}$ approaches are much less than 5\%. Conversely, the VaR based on the VC and MC approaches are less than EVT-VaR when the confidence level is 99\%. This phenomenon may be caused by the fat tails of WTI and Brent returns.

(3) No matter for upside or downside, the VaR of WTI returns is larger than that of Brent returns at identical confidence levels. It demonstrates that WTI crude oil market has more extreme risk compared to Brent market.

(4) The GED-GARCH model can estimate the downside dynamic VaR more accurately than the upside dynamic VaR for the Brent and WTI markets. This may result from the fact that the returns in the two oil markets have fat tails and leptokurtic distributions.

However, there are also several limitations to our methods. With respect to future work, there are several aspects to be conducted further. First of all, the selection of the threshold in the EVT method is controversial. This study uses the Mean Excess Function to determine the threshold. Other methods should be developed to gain more objective and reasonable assessment. Second, we need to pay attention to the forecasting of future risk in oil markets. For this end, the whole sample period can be divided into two parts. The first sample period is applied to measure the VaR, and the other one is used for forecasting purposes. In addition, the GED-GARCH model fails to measure the upside dynamic VaR during the sample period when the confidence level is $95 \%$, so some more attention can be paid to the estimation of upside dynamic VaR in oil market.

\section{Acknowledgements}

The authors gratefully acknowledge the financial support of National Strategic Research Program 
of China (2016YFA0602600), National Natural Science Foundation of China (71020107026, 71573013 and 41328008), the UK Economic and Social Research Council (ES/L016028/1) Natural Environment Research Council (NE/N00714X/1) and British Academy Grant (AF150310).

\section{References}

Aloui, Chaker, and Samir Mabrouk. 2010. "Value-at-risk estimations of energy commodities via long-memory, asymmetry and fat-tailed GARCH models." Energy Policy 38 (5): 2326-2339. doi: 10.1016/j.enpol.2009.12.020.

Balkema, August A, and Laurens De Haan. 1974. "Residual life time at great age." The Annals of Probability 2: 792-804.

Baumol, William J. 1963. "An expected gain-confidence limit criterion for portfolio selection." Management Science 10 (1): 174-182. doi: 10.1287/mnsc.10.1.174.

Bianconi, Marcelo, and Joe A. Yoshino. 2014. "Risk factors and value at risk in publicly traded companies of the nonrenewable energy sector." Energy Economics 45: 19-32. doi: 10.1016/j.eneco.2014.06.018.

Bollerslev, Tim. 1986. "Generalized autoregressive conditional heteroskedasticity." Journal of Econometrics 31 (3): 307-327. doi: 10.1016/0304-4076(86)90063-1.

Boroumand, Raphaël Homayoun, and Georg Zachmann. 2012. "Retailers' risk management and vertical arrangements in electricity markets." Energy Policy 40: 465-472. doi: 10.1016/j.enpol.2011.10.041.

Cabedo, J. David, and Ismael Moya. 2003. "Estimating oil price 'Value at Risk' using the historical simulation approach." Energy Economics 25 (3): 239-253. doi: 10.1016/S0140-9883(02)00111-1.

Chang, Kuang-Liang. 2011. "The optimal value-at-risk hedging strategy under bivariate regime switching ARCH framework." Applied Economics 43 (21): 2627-2640. doi: $10.1080 / 00036840903299771$.

Christoffersen, Peter F. 1998. "Evaluating interval forecasts." International Economic Review 39 (4): 841-862. doi: 10.2307/2527341.

Christoffersen, Peter, and Denis Pelletier. 2004. "Backtesting value-at-risk: A duration-based 
approach." Journal of Financial Econometrics 2 (1): 84-108. doi: 10.1093/jjfinec/nbh004.

Chuang, Chung-Chu, Yi-Hsien Wang, Tsai-Jung Yeh, and Shuo-Li Chuang. 2015. "Hedging effectiveness of the hedged portfolio: the expected utility maximization subject to the value-at-risk approach." Applied Economics 47 (20): 2040-2052. doi: 10.1080/00036846.2014.1000528.

Cong, Rong-Gang, and Shaochuan Shen. 2013. "Relationships among energy price shocks, stock market, and the macroeconomy: Evidence from China." The Scientific World Journal 2013: 1-9. doi: $10.1155 / 2013 / 171868$

Cong, Rong-Gang, Yi-Ming Wei, Jian-Lin Jiao, and Ying Fan. 2008. "Relationships between oil price shocks and stock market: An empirical analysis from China." Energy Policy 36 (9): 3544-3553. doi: 10.1016/j.enpol.2008.06.006.

Duffie, Darrell, and Jun Pan. 1997. "An overview of value at risk." The Journal of Derivatives 4 (3): 7-49. doi: 10.3905/jod.1997.407971.

Embrechts, Paul, Claudia Klüppelberg, and Thomas Mikosch. 1997. Modelling extremal events: For insurance and finance. Berlin: Springer.

Ergen, Ibrahim. 2014. "Tail dependence and diversification benefits in emerging market stocks: an extreme value theory approach." Applied Economics 46 (19): 2215-2227. doi: $10.1080 / 00036846.2014 .899678$.

Fan, Ying, Yue-Jun Zhang, Hsien-Tang Tsai, and Yi-Ming Wei. 2008. "Estimating 'Value at Risk' of crude oil price and its spillover effect using the GED-GARCH approach." Energy Economics 30 (6): 3156-3171. doi: 10.1016/j.eneco.2008.04.002.

Fretheim, Torun, and Glenn Kristiansen. 2015. "Commodity market risk from 1995 to 2013: an extreme value theory approach." Applied Economics 47 (26): 2768-2782. doi: $10.1080 / 00036846.2015 .1011307$

Giot, Pierre, and Sébastien Laurent. 2003. "Market risk in commodity markets: A VaR approach." Energy Economics 25 (5): 435-457. doi: 10.1016/S0140-9883(03)00052-5.

He, Kaijian, Kin Keung Lai, and Guocheng Xiang. 2012. "Portfolio Value at Risk estimate for crude oil markets: A multivariate wavelet denoising approach." Energies 5 (4): 1018-1043. doi: 10.3390/en5041018.

Huang, Alex YiHou. 2015. "Value at risk estimation by threshold stochastic volatility model." Applied Economics 47 (45): 4884-4900. doi: 10.1080/00036846.2015.1037439. 
Ji, Qiang, and Jian-Feng Guo. 2015. "Oil price volatility and oil-related events: An Internet concern study perspective." Applied Energy 137: 256-264. doi: 10.1016/j.apenergy.2014.10.002.

Katz, Richard W, Marc B Parlange, and Philippe Naveau. 2002. "Statistics of extremes in hydrology." Advances in Water Resources 25 (8): 1287-1304. doi: 10.1016/S0309-1708(02)00056-8.

Kupiec, Paul H. 1995. "Techniques for verifying the accuracy of risk measurement models." The Journal of Derivatives 3 (2): 73-84.

Leccadito, Arturo, Simona Boffelli, and Giovanni Urga. 2014. "Evaluating the accuracy of value-at-risk forecasts: New multilevel tests." International Journal of Forecasting 30 (2): 206-216. doi: 10.1016/j.ijforecast.2013.07.014.

Lee, Ming-Chih, Chien-Liang Chiu, and Wan-Hsiu Cheng. 2010. "Modeling Value-at-Risk in oil price using bootstrapping approach." International Research Journal of Finance and Economics 40: $7-19$.

Marimoutou, Velayoudoum, Bechir Raggad, and Abdelwahed Trabelsi. 2009. "Extreme value theory and value at risk: Application to oil market." Energy Economics 31 (4): 519-530. doi: 10.1016/j.eneco.2009.02.005.

McNeil, Alexander J. 1997. "Estimating the tails of loss severity distributions using extreme value theory." Astin Bulletin 27 (1): 117-137. doi: 10.2143/AST.27.1.563210.

McNeil, Alexander J, and Rüdiger Frey. 2000. "Estimation of tail-related risk measures for heteroscedastic financial time series: An extreme value approach." Journal of Empirical Finance 7 (3): 271-300. doi: 10.1016/S0927-5398(00)00012-8.

Mi, Zhi-Fu, and Yue-Jun Zhang. 2011. "Estimating the 'value at risk' of EUA futures prices based on the extreme value theory." International Journal of Global Energy Issues 35 (2): 145-157. doi: 10.1504/IJGEI.2011.045027.

Nelson, Daniel B. 1990. "ARCH models as diffusion approximations." Journal of Econometrics 45 (1): 7-38. doi: 10.1016/0304-4076(90)90092-8.

Nomikos, Nikos K., and Panos K. Pouliasis. 2011. "Forecasting petroleum futures markets volatility: The role of regimes and market conditions." Energy Economics 33 (2): 321-337. doi: 10.1016/j.eneco.2010.11.013.

Parkinson, Aidan, and Peter Guthrie. 2014. "Evaluating the energy performance of buildings within a value at risk framework with demonstration on UK offices." Applied Energy 133: 40-55. doi: 
10.1016/j.apenergy.2014.07.074.

Pickands, James. 1975. "Statistical inference using extreme order statistics." Annals of Statistics 3: 119-131.

Reboredo, Juan C., and Miguel A. Rivera-Castro. 2014a. "Gold and exchange rates: Downside risk and hedging at different investment horizons." International Review of Economics \& Finance 34: 267-279. doi: 10.1016/j.iref.2014.07.005.

Reboredo, Juan C., and Miguel A. Rivera-Castro. 2014b. "Wavelet-based evidence of the impact of oil prices on stock returns." International Review of Economics \& Finance 29: 145-176. doi: 10.1016/j.iref.2013.05.014.

Sadeghi, Mehdi, and Saeed Shavvalpour. 2006. "Energy risk management and value at risk modeling." Energy Policy 34 (18): 3367-3373. doi: 10.1016/j.enpol.2005.07.004.

Silva Filho, Osvaldo C., Flavio A. Ziegelmann, and Michael J. Dueker. 2014. "Assessing dependence between financial market indexes using conditional time-varying copulas: applications to Value at Risk (VaR)." Quantitative Finance 14 (12): 2155-2170. doi: 10.1080/14697688.2012.739726.

Su, Jung-Bin. 2014. "How to mitigate the impact of inappropriate distributional settings when the parametric value-at-risk approach is used." Quantitative Finance 14 (2): 305-325. doi: 10.1080/14697688.2012.738934.

Ze-To, Samuel Yau Man. 2012. "Estimating value-at-risk under a Heath-Jarrow-Morton framework with jump." Applied Economics 44 (21): 2729-2741. doi: 10.1080/00036846.2011.566198.

Zhang, Yue-Jun, and Yi-Ming Wei. 2010. "The crude oil market and the gold market: Evidence for cointegration, causality and price discovery." Resources Policy 35 (3): 168-177. doi: 10.1016/j.resourpol.2010.05.003.

Zhang, Yue-Jun, and Yi-Ming Wei. 2011. "The dynamic influence of advanced stock market risk on international crude oil returns: an empirical analysis." Quantitative Finance 11 (7): 967-978. doi: 10.1080/14697688.2010.538712. 
Table 1. Summary statistics of oil price returns in the WTI and Brent markets.

\begin{tabular}{cccccccc}
\hline Return & Mean & Standard Deviations & Max & Min & Skewness & Kurtosis & JB test \\
\hline WTI & 0.0146 & 2.4102 & 18.8677 & -40.6396 & -0.8473 & 16.8603 & 84447.65 \\
& & & & & & & \\
& & & & & & & \\
Brent & 0.0156 & 2.2480 & 18.1297 & -36.1214 & -0.6771 & 15.7323 & 73329.89 \\
& & & & & & & \\
& & & & & & & $(0.0000)$ \\
\hline
\end{tabular}

Note: The $p$-value is presented in the parenthesis. 
Table 2. Static VaR estimation results at the confidence level of $95 \%$.

\begin{tabular}{|c|c|c|c|c|c|c|c|}
\hline Return & Risk type & Approach & VaR & Observations & Failure time & Failure rate & $L R$ \\
\hline \multirow{6}{*}{ WTI } & \multirow{3}{*}{ Upside } & EVT & 3.47 & 7069 & 344 & $4.87 \%$ & 0.27 \\
\hline & & $\mathrm{VC}$ & 3.98 & 7069 & 252 & $3.56 \%$ & 33.92 \\
\hline & & $\mathrm{MC}$ & 3.99 & 7069 & 249 & $3.52 \%$ & 36.07 \\
\hline & \multirow{3}{*}{ Downside } & EVT & 3.61 & 7069 & 353 & $4.99 \%$ & 0.00 \\
\hline & & $\mathrm{VC}$ & 3.95 & 7069 & 279 & $3.95 \%$ & 17.74 \\
\hline & & $\mathrm{MC}$ & 3.93 & 7069 & 287 & $4.06 \%$ & 14.01 \\
\hline \multirow{6}{*}{ Brent } & \multirow{3}{*}{ Upside } & EVT & 3.36 & 7069 & 350 & $4.95 \%$ & 0.04 \\
\hline & & $\mathrm{VC}$ & 3.71 & 7069 & 256 & $3.62 \%$ & 31.15 \\
\hline & & $\mathrm{MC}$ & 3.73 & 7069 & 252 & $3.56 \%$ & 33.92 \\
\hline & \multirow{3}{*}{ Downside } & EVT & 3.48 & 7069 & 338 & $4.78 \%$ & 0.72 \\
\hline & & $\mathrm{VC}$ & 3.68 & 7069 & 293 & $4.14 \%$ & 11.53 \\
\hline & & $\mathrm{MC}$ & 3.72 & 7069 & 283 & $4.00 \%$ & 15.82 \\
\hline
\end{tabular}


Table 3. Static VaR estimation results at the confidence level of $99 \%$.

\begin{tabular}{|c|c|c|c|c|c|c|c|}
\hline Return & Risk type & Approach & VaR & Observations & Failure time & Failure rate & $L R$ \\
\hline \multirow{6}{*}{ WTI } & \multirow{3}{*}{ Upside } & EVT & 6.22 & 7069 & 69 & $0.98 \%$ & 0.04 \\
\hline & & $\mathrm{VC}$ & 5.62 & 7069 & 90 & $1.27 \%$ & 4.90 \\
\hline & & $\mathrm{MC}$ & 5.58 & 7069 & 92 & $1.30 \%$ & 5.93 \\
\hline & \multirow{3}{*}{ Downside } & EVT & 6.70 & 7069 & 72 & $1.02 \%$ & 0.02 \\
\hline & & $\mathrm{VC}$ & 5.59 & 7069 & 120 & $1.70 \%$ & 28.73 \\
\hline & & $\mathrm{MC}$ & 5.61 & 7069 & 119 & $1.68 \%$ & 27.67 \\
\hline \multirow{6}{*}{ Brent } & \multirow{3}{*}{ Upside } & EVT & 5.78 & 7069 & 76 & $1.08 \%$ & 0.39 \\
\hline & & $\mathrm{VC}$ & 5.24 & 7069 & 100 & $1.41 \%$ & 10.88 \\
\hline & & $\mathrm{MC}$ & 5.24 & 7069 & 100 & $1.41 \%$ & 10.88 \\
\hline & \multirow{3}{*}{ Downside } & EVT & 5.79 & 7069 & 75 & $1.06 \%$ & 0.26 \\
\hline & & $\mathrm{VC}$ & 5.21 & 7069 & 96 & $1.36 \%$ & 8.23 \\
\hline & & $\mathrm{MC}$ & 5.28 & 7069 & 91 & $1.29 \%$ & 5.40 \\
\hline
\end{tabular}


Table 4. Estimation results of GARCH models for WTI and Brent returns.

\begin{tabular}{lll}
\hline Parameter & WTI & Brent \\
\hline$\alpha_{0}$ & $0.0512(0.0000)$ & $0.0298(0.0000)$ \\
$\alpha_{1}$ & $0.0688(0.0000)$ & $0.0658(0.0000)$ \\
$\beta_{1}$ & $0.9234(0.0000)$ & $0.9301(0.0000)$ \\
AIC value & 4.2505 & 4.1227 \\
Log likelihood & -15015.12 & -14563.80 \\
GED degrees of freedom & $1.3235(0.0000)$ & $1.3525(0.0000)$ \\
\hline
\end{tabular}

Note: The $p$-value is presented in the parenthesis. 
Table 5. Summary of dynamic VaR of WTI and Brent returns.

\begin{tabular}{|c|c|c|c|c|c|c|c|c|c|}
\hline Return & $\begin{array}{c}\text { confide } \\
\text { nce } \\
\text { level }\end{array}$ & Risk type & Mean & $\begin{array}{c}\text { Standard } \\
\text { deviation } \\
\mathrm{s}\end{array}$ & Max & Min & $\begin{array}{c}\text { Failure } \\
\text { time }\end{array}$ & $\begin{array}{c}\text { Failure } \\
\text { rate }\end{array}$ & $L R$ \\
\hline \multirow{4}{*}{ WTI } & \multirow{2}{*}{$95 \%$} & Upside & 3.7072 & 1.6157 & 19.8443 & 1.6852 & 270 & $3.82 \%$ & 22.50 \\
\hline & & Downside & -3.6190 & 1.6112 & -1.6191 & -18.6567 & 353 & $4.99 \%$ & 0.00 \\
\hline & \multirow{2}{*}{$99 \%$} & Upside & 5.7664 & 2.5217 & 30.4351 & 2.6178 & 48 & $0.68 \%$ & 8.29 \\
\hline & & Downside & -5.6782 & 2.5173 & -2.5489 & -28.9821 & 87 & $1.23 \%$ & 3.54 \\
\hline \multirow{4}{*}{ Brent } & \multirow{2}{*}{$95 \%$} & Upside & 3.4827 & 1.5085 & 18.1832 & 0.6375 & 272 & $3.85 \%$ & 21.39 \\
\hline & & Downside & -3.3997 & 1.5132 & -0.6242 & -20.0207 & 374 & $5.29 \%$ & 1.24 \\
\hline & \multirow{2}{*}{$99 \%$} & Upside & 5.3854 & 2.3420 & 28.4090 & 0.9862 & 58 & $0.82 \%$ & 2.45 \\
\hline & & Downside & -5.3025 & 2.3468 & -0.9730 & -30.4084 & 77 & $1.09 \%$ & 0.55 \\
\hline
\end{tabular}




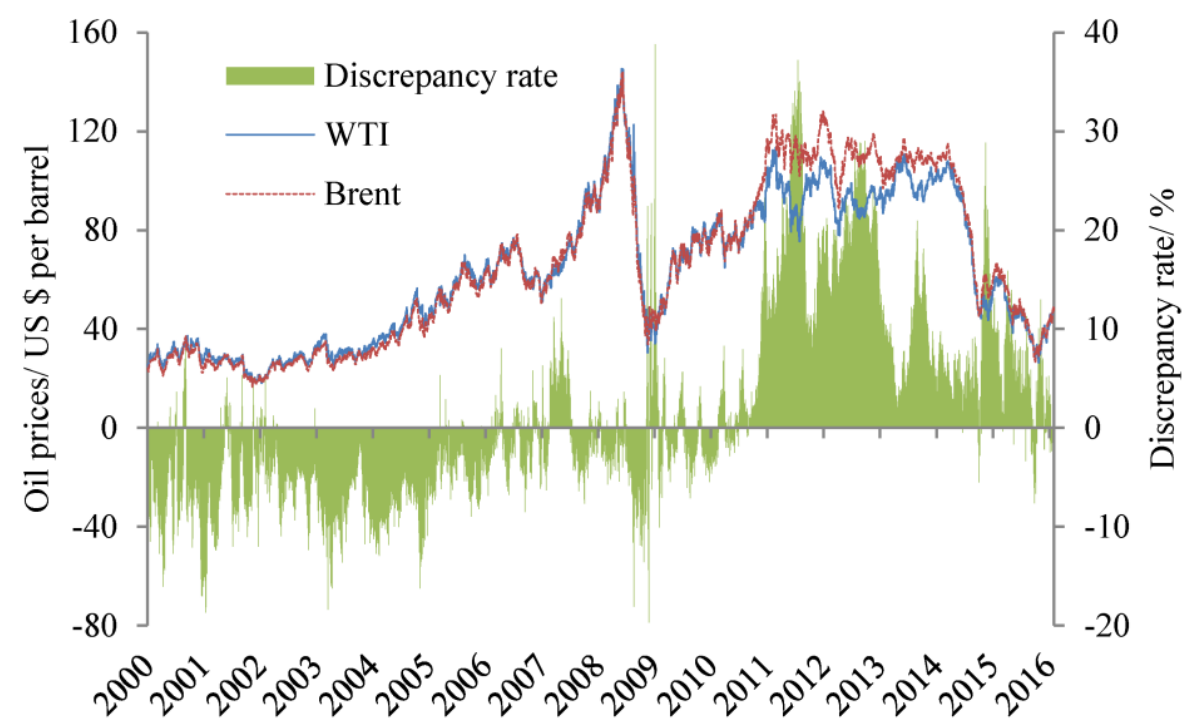

Figure 1. Daily spot prices and discrepancy rates of WTI and Brent crude oil. Discrepancy rate = 100* (Brent-WTI)/WTI. 


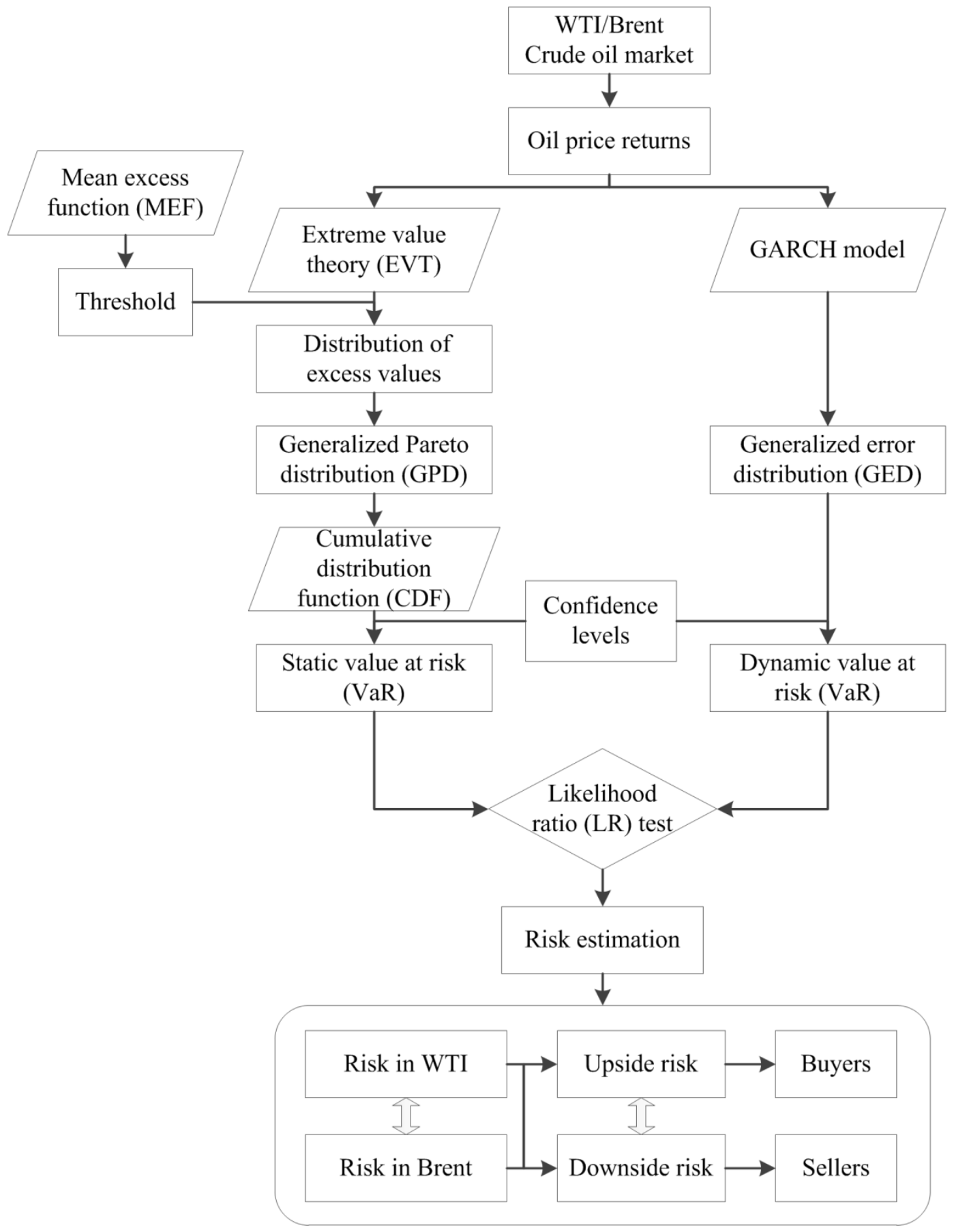

Figure 2. Framework of VaR estimation using the EVT-GED-GARCH integrated model. 

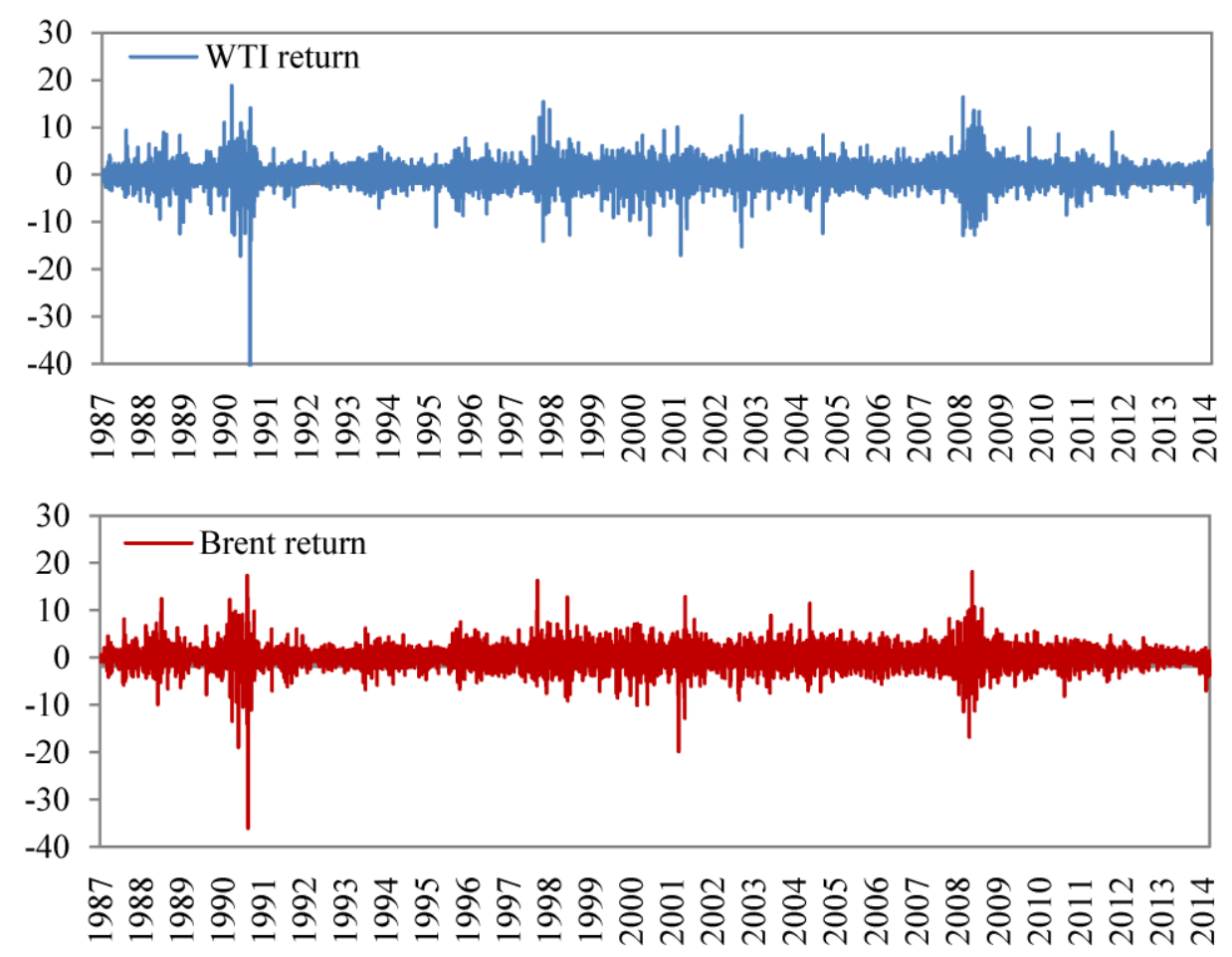

Figure 3. Daily price returns of WTI and Brent crude oil. 


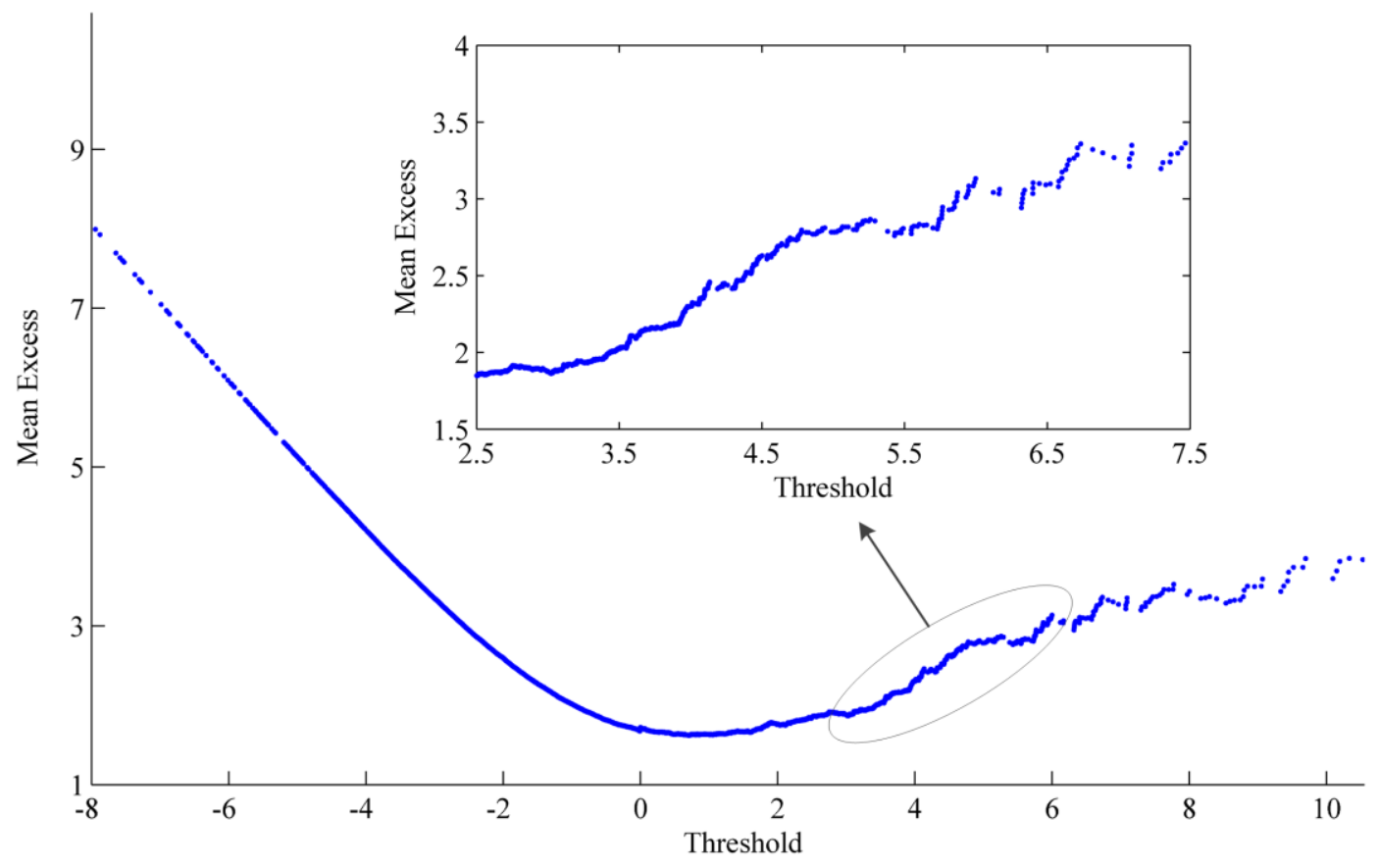

Figure 4. The MEF plot of WTI loss series. 


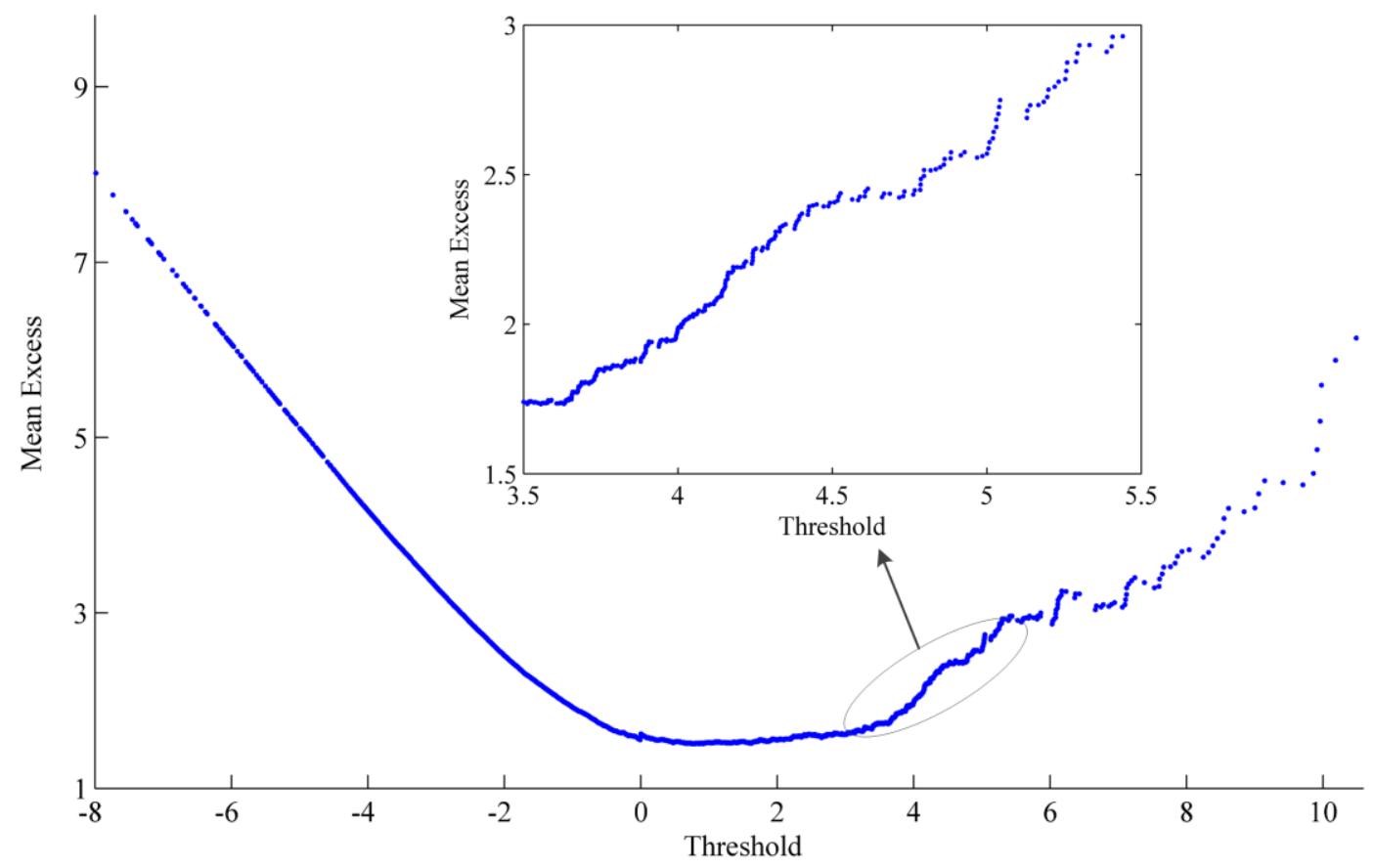

Figure 5. The MEF plot of Brent loss series. 


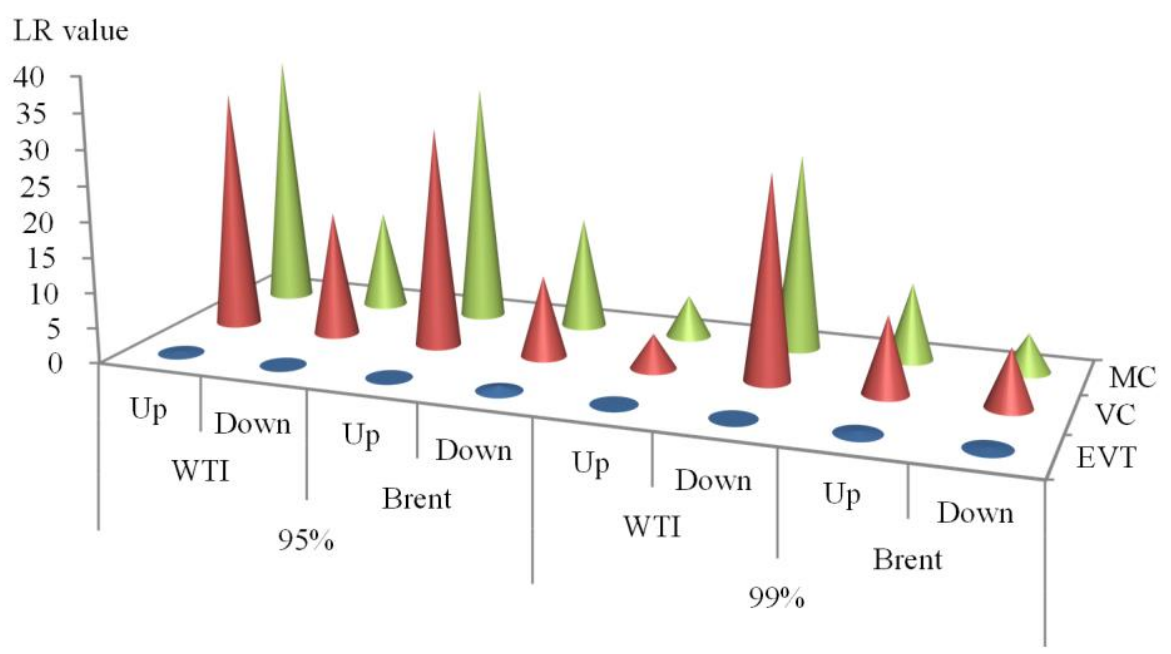

Figure 6. The $L R$ values of three VaR estimation methods. 


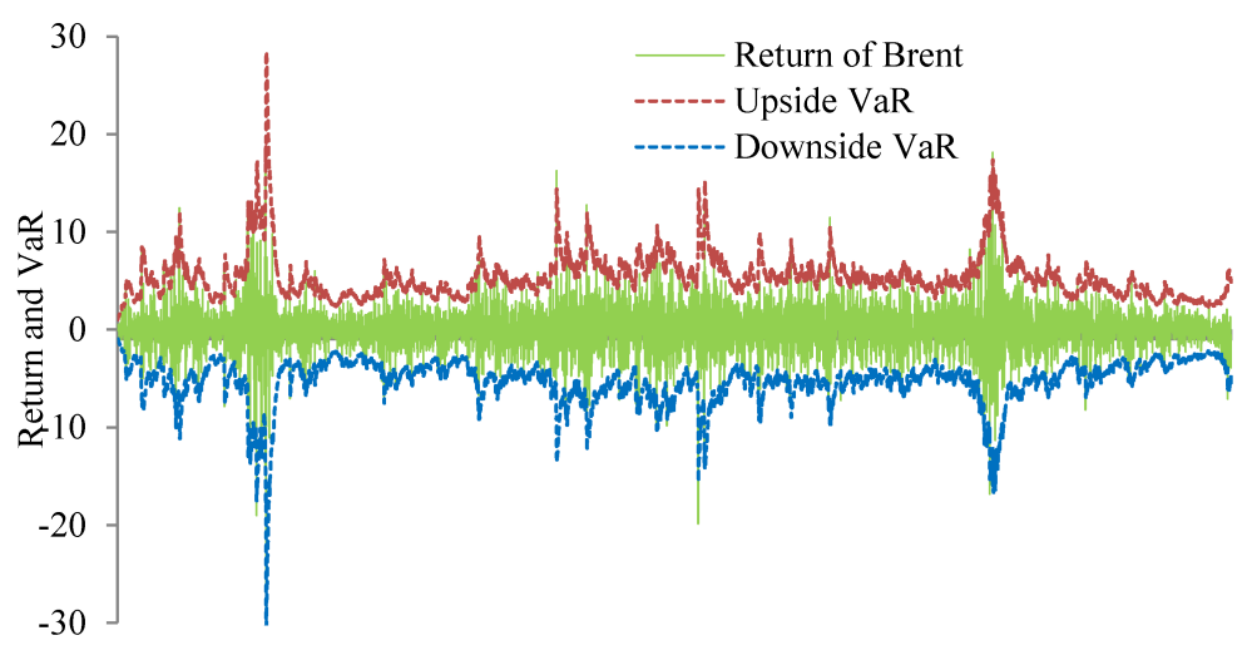

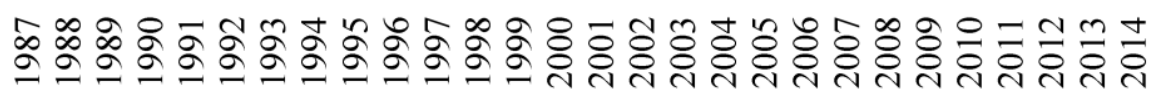

Figure 7. Performance of GED-GRACH based VaR for Brent returns at the $99 \%$ confidence level. 